\title{
Enhancement of the Immunostimulatory Effect of Phosphodiester CpG Oligodeoxynucleotides by an Antiparallel Guanine- Quadruplex Structural Scaffold
}

\author{
Fika Ayu Safitri ${ }^{1,2}$, Anh Thi Tram Tu ${ }^{2,3}{ }^{(D}$, Kazuaki Hoshi ${ }^{2}$, Miwako Shobo ${ }^{2}$, Dandan Zhao ${ }^{2}$, Arief Budi Witarto ${ }^{4}$, \\ Sony Heru Sumarsono ${ }^{5}$, Ernawati Arifin Giri-Rachman ${ }^{5}$, Kaori Tsukakoshi ${ }^{6}$, Kazunori Ikebukuro ${ }^{6}$ (D)
} and Tomohiko Yamazaki ${ }^{2,3, * \mathbb{D}}$

check for updates

Citation: Safitri, F.A.; Tu, A.T.T.; Hoshi, K.; Shobo, M.; Zhao, D.; Witarto, A.B.; Sumarsono, S.H.; Giri-Rachman, E.A.; Tsukakoshi, K.; Ikebukuro, K.; et al. Enhancement of the Immunostimulatory Effect of Phosphodiester CpG

Oligodeoxynucleotides by an Antiparallel Guanine-Quadruplex Structural Scaffold. Biomolecules 2021, 11, 1617. https://doi.org/10.3390/ biom 11111617

Academic Editor: Paolo Fagone

Received: 25 September 2021 Accepted: 27 October 2021

Published: 1 November 2021

Publisher's Note: MDPI stays neutral with regard to jurisdictional claims in published maps and institutional affiliations.

Copyright: (c) 2021 by the authors Licensee MDPI, Basel, Switzerland. This article is an open access article distributed under the terms and conditions of the Creative Commons Attribution (CC BY) license (https:/ / creativecommons.org/licenses/by/ $4.0 /)$.
1 Doctoral Program in Biology, School of Life Sciences and Technology, Institut Teknologi Bandung (ITB), Bandung 40132, West Java, Indonesia; fikaayusafitri1904@gmail.com

2 Research Center for Functional Materials, National Institute for Materials Science, 1-2-1, Sengen, Tsukuba 305-0047, Japan; TU.ThiTramAnh@nims.go.jp (A.T.T.T.); k.hoshi.oe@juntendo.ac.jp (K.H.); SHOBO.Miwako@nims.go.jp (M.S.);ZHAO.Dandan@nims.go.jp (D.Z.)

3 Division of Life Science, Graduate School of Life Science, Hokkaido University, Kita 10, Nishi 8, Kita-ku, Sapporo 060-0808, Japan

4 Department of Molecular Biology and Biochemistry, Faculty of Medicine, Indonesia Defense University, Bogor 16810, West Java, Indonesia; arief.witarto@idu.ac.id

5 Physiology, Developmental Biology and Biomedical Sciences Research Group, School of Life Sciences and Technology, ITB, Bandung 40132, West Java, Indonesia; sonyheru@sith.itb.ac.id (S.H.S.); erna@sith.itb.ac.id (E.A.G.-R.)

6 Department of Biotechnology and Life Science, Tokyo University of Agriculture and Technology, 2-24-16, Naka-cho, Koganei 184-8588, Japan; k-tsuka@cc.tuat.ac.jp (K.T.); ikebu@cc.tuat.ac.jp (K.I.)

* Correspondence: YAMAZAKI.Tomohiko@nims.go.jp; Tel.: +81-29-859-2345; Fax: +81-29-859-2449

\begin{abstract}
Guanine-quadruplex-based CpG oligodeoxynucleotides (G4 CpG ODNs) have been developed as potent immunostimulatory agents with reduced sensitivity to nucleases. We designed new monomeric G4 ODNs with an antiparallel topology using antiparallel type duplex/G4 ODNs as robust scaffolds, and we characterized their topology and effects on cytokine secretion. Based on circular dichroism analysis and quantification of mRNA levels of immunostimulatory cytokines, it was found that monomeric antiparallel G4 CpG ODNs containing two CpG motifs in the first functional loop, named G2.0.0, could maintain antiparallel topology and generate a high level of immunostimulatory cytokines in RAW264 mouse macrophage-like cell lines. We also found that the flanking sequence in the CpG motif altered the immunostimulatory effects. Gc2c.0.0 and Ga2c.0.0 are monomeric antiparallel G4 CpG ODNs with one cytosine in the $3^{\prime}$ terminal and one cytosine/adenine in the $5^{\prime}$ terminal of $\mathrm{CpG}$ motifs that maintained the same resistance to degradation in serum as G2.0.0 and improved interleukin-6 production in RAW264 and bone marrow-derived macrophages. The immunostimulatory activity of antiparallel G4 CpG ODNs is superior to that of linear natural CpG ODNs. These results provide insights for the rational design of highly potent CpG ODNs using antiparallel G4 as a robust scaffold.
\end{abstract}

Keywords: guanine quadruplex; high-order structure; monomeric; immunostimulatory activity; adjuvant

\section{Introduction}

Synthetic oligodeoxynucleotides containing CpG motifs (CpG ODNs) are short segments of DNA that represent a type of pathogen-associated molecular pattern that can stimulate immune cells expressing toll-like receptor 9 (TLR9), such as dendritic cells and macrophages, and activate the immune response [1,2]. The initial recognition of $\mathrm{CpG}$ ODNs by the leucine-rich repeat in the extracellular domain of TLR9 leads to the dimerization of TLR9 that initiates an immune response by recruiting the MyD88-IRAK adaptor, resulting in the activation of multiple transcription factors, including NF- $\mathrm{KB}, \mathrm{AP}-1$, and 
IRF-7 [1]. Currently, synthetic CpG ODNs have great promise as vaccine adjuvants [2], immunotherapy agents for allergy [3], and anti-cancer agents [4]. However, ODNs with a phosphodiester (PD) backbone are susceptible to nuclease degradation, thus limiting their clinical application. Phosphorothioate-modified CpG ODNs have been well-studied as an alternative to enhance resistance against nucleases and are already utilized as vaccine adjuvants for the hepatitis B vaccine (HEPLISAV-B). However, phosphorothioate modification is associated with undesirable side effects, such as prolonged coagulation time, acute toxicity, and non-specific binding to proteins, raising concerns regarding its safety [5-8].

Non-canonical guanine-quadruplex (G4) structures can increase the nuclease resistance of CpG ODNs with a PD backbone [9-11]. The hybrid type G4 CpG ODNs with CpG motif in the central loop showed high immunostimulatory effect with acquired high nuclease resistance and cellular uptake [9]. However, the G4 structure frequently changes depending on the surrounding environment, including the type and concentration of coordinating metal cations (potassium, sodium, etc.) and the sequence and length of the loop regions [12,13]. Modifying the CpG ODNs in the G4 structure poses a challenge for assembly because of such changes in the G4 structure. Since the topology of G4 CpG ODNs changes depending on the length and sequence introduced into the loop region, it is difficult to accurately design G4 CpG ODNs. We aim to enhance the nuclease resistance and cellular uptake using the G4 structure, and it is important to control the topology of G4 structures for future rational design. To overcome the challenge of structural changes in G4, we used a well-known unimolecular quadruplex as a scaffold for CpG ODNs. We employed an antiparallel duplex/G4 DNA aptamer, RE31, which is a thrombin aptamer consisting of a 31-mer ODN that forms a unimolecular quadruplex comprising a stack of two guanine quartets (hereafter referred to as G-tetrad) connected by two external loops and one central loop with a 6-mer duplex region, as our basic design. RE31 shows high thrombin-inhibitory activity against bovine thrombin [14], human thrombin, and rat thrombin [15] and has a high affinity for thrombin and prothrombin [16]. RE31 shows a typical antiparallel circular dichroism (CD) spectrum with two positive peaks around $295 \mathrm{~nm}$ and $245 \mathrm{~nm}$ and a negative peak at $265 \mathrm{~nm}$. The three-dimensional structure of RE31 has been previously determined [17]. Therefore, we designed a new high-order structured CpG ODN by introducing a CpG motif in the loop of RE31 while maintaining the anti-parallel topology of RE31.

In this study, we developed new monomeric G4 ODNs with an anti-parallel topology using the structurally analyzed duplex/G4 DNA, RE31, as a robust scaffold. The objective of this study was to maintain antiparallel duplex/G4 ODN structure and its stability when CpG motif was introduced to increase immunostimulatory activity. This finding provides a basis for future rational design of G4 CpG ODNS for vaccine adjuvant applications.

\section{Materials and Methods}

\subsection{G4 Formation of $C p G$ ODNs}

HPLC-grade ODNs with PD backbones (Table 1) were purchased from Eurofins Genomics (Tokyo, Japan). The ODNs were dissolved in sterile Milli-Q water to prepare a stock solution of approximately $100 \mu \mathrm{M}$. The concentration of ODNs was quantified using a NanoDrop 2000 Spectrophotometer (Thermo Fisher Scientific, Waltham, MA, USA). In Gquadruplex formation, the stocked ODNs were diluted in Dulbecco's phosphate-buffered saline (D-PBS; DS Pharma Biomedical, Osaka, Japan), heated at $95^{\circ} \mathrm{C}$ for $5 \mathrm{~min}$, followed by slow cooling to $4{ }^{\circ} \mathrm{C}$ for $30 \mathrm{~min}$, and then stored at $4{ }^{\circ} \mathrm{C}$ for further experiments.

\section{2. $C D$ Measurement}

To measure the CD spectrum, $2 \mu \mathrm{M}$ CpG ODNs were dispensed into a 1-cm path length quartz cuvette (model T-18-ES-10, $1 \mathrm{~cm}$ path length, JASCO) and analyzed using a JASCO J-1500 spectropolarimeter at a scan speed of $50 \mathrm{~nm} / \mathrm{min}$, response time of $2.0 \mathrm{~s}$, bandwidth of $1.0 \mathrm{~nm}$, resolution of $0.2 \mathrm{~nm}$, and sensitivity of 100 millidegrees (mdeg). $\mathrm{CD}$ spectra were recorded as the average of five replications over the spectral range of 
220-320 nm at $10^{\circ} \mathrm{C}$ or $25^{\circ} \mathrm{C}$. The D-PBS buffer spectrum was measured under the same conditions and subtracted as the background from each spectrum.

Table 1. Sequences of loop modification oligonucleotides in this study.

\begin{tabular}{|c|c|c|}
\hline Name & Sequence $5^{\prime}$ to $3^{\prime}$ & Length \\
\hline G0.0.0 & GTGACGTAGGTTGGTGTGGTTGGGGCGTCAC & 31 \\
\hline G2.0.0 & GTGACGTAGGGTCGTTTTGTCGTTGGTGTGGTTGGGGCGTCAC & 43 \\
\hline G0.2.0 & GTGACGTAGGTTGGGTCGTTTTGTCGTTGGTTGGGGCGTCAC & 42 \\
\hline G0.0.2 & GTGACGTAGGTTGGTGTGGGTCGTTTTGTCGTTGGGGCGTCAC & 43 \\
\hline G1.0.0 & GTGACGTAGGGTCGTTGGTGTGGTTGGGGCGTCAC & 35 \\
\hline Ga2a.0.0 & GTGACGTAGGAGTCGTTTTGTCGTTAGGTGTGGTTGGGGCGTCAC & 45 \\
\hline Gc2c.0.0 & GTGACGTAGGCGTCGTTTTGTCGTTCGGTGTGGTTGGGGCGTCAC & 45 \\
\hline Ga2c.0.0 & 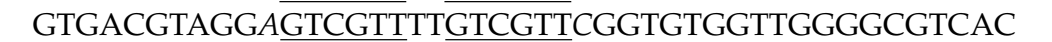 & 45 \\
\hline Gc2a.0.0 & GTGACGTAGGCGTCGTTTTGTCGTTAGGTGTGGTTGGGGCGTCAC & 45 \\
\hline Gac2ac. 0.0 & GTGACGTAGGACGTCGTTTTGTCGTTACGGTGTGGTTGGGGCGTCAC & 47 \\
\hline Gac2ca.0.0 & GTGACGTAGGACGTCGTTTTGTCGTTCAGGTGTGGTTGGGGCGTCAC & 47 \\
\hline Gca2ac. 0.0 & GTGACGTAGGCAGTCGTTTTGTCGTT ACGGTGTGGTTGGGGCGTCAC & 47 \\
\hline Gca2ca.0.0 & GTGACGTAGGACGTCGTTTTGTCGTTCAGGTGTGGTTGGGGCGTCAC & 47 \\
\hline Linear $2 \mathrm{CpG}$ & GTCGTTTTGTCGTT & \\
\hline
\end{tabular}

Note: CpG motifs are underlined. All ODNs have a phosphodiester backbone.

The CD melting experiments were performed from $5{ }^{\circ} \mathrm{C}$ to $90{ }^{\circ} \mathrm{C}$ with a temperature gradient of $1^{\circ} \mathrm{C} / \mathrm{min}$ based on the measurement of absorbance at $295 \mathrm{~nm}$, as previously reported [9]. The Tm value was calculated by determining the upper and lower baseline values [18]. Linear regression was performed using GraphPad Prism 8 version 8.2.0 for Windows (GraphPad Software, La Jolla, CA, USA).

\subsection{Polyacrylamide Gel Electrophoresis (PAGE)}

To analyze the structure of the ODNs, $2 \mu \mathrm{L}$ of $0.5 \mu \mathrm{M}$ ODNs were applied to a $12 \%$ polyacrylamide gel (PAGE; $1 \mathrm{~mm}$ thick; TEFCO; Tokyo, Japan). Electrophoresis was performed at $4{ }^{\circ} \mathrm{C}$ at a constant voltage $(180 \mathrm{~V})$ in $0.5 \times$ Tris-borate-EDTA (TBE) buffer (0.089 M, pH 8.3-8.5; Takara Bio, Shiga, Japan) supplemented with 4 mM KCl. A 10-base pair (bp) DNA ladder (Thermo Fisher Scientific) was used as a marker. The DNA in the gel was stained with SYBR Gold Nucleic Acid Gel stain (Thermo Fisher Scientific). Linear ODNs were used as references.

\subsection{Stability of ODNs in Serum}

The stability of the ODNs in serum was analyzed by adding $36 \mu \mathrm{L}$ of $20 \%$ fetal bovine serum (FBS) into a tube, followed by the addition of $4 \mu \mathrm{L}$ of $10 \mu \mathrm{M}$ ODNs. The tube was incubated at $37^{\circ} \mathrm{C}$ for $1,2,4$, and $24 \mathrm{~h}$. After the incubation, $4 \mu \mathrm{L}$ of $250 \mathrm{mM}$ EDTA was added to stop the reaction, and the tube was heated for $2 \mathrm{~min}$ at $80^{\circ} \mathrm{C}$. The ODNs were stored at $4{ }^{\circ} \mathrm{C}$ until PAGE analysis. Serum-treated ODNs were separated using e-PAGEL gel (15\%, ATTO, Tokyo, Japan) in $0.5 \times$ TBE buffer containing $4 \mathrm{mM} \mathrm{KCl}$ at a constant current of $21 \mathrm{~mA}$ at $4^{\circ} \mathrm{C}$. Quantification of the undegraded ODNs was performed using the Image Studio Lite software (LI-COR Biotechnology, Lincoln, NE, USA) by determining the fluorescence intensity of the corresponding bands stained with SYBR Gold.

\subsection{Cell Culture}

The mouse macrophage-like RAW264 cell line (RCB0535) was purchased from RIKEN BioResource Center (Tsukuba, Japan) and maintained in minimum essential medium (MEM) (Gibco, Carlsbad, CA, USA) supplemented with 10\% $(v / v)$ heat-inactivated FBS (Sigma-Aldrich, St Louis, MO, USA) and 1\% (v/v) non-essential amino acid solution (NEAA, 100X, Wako Pure Chemical Industries, Osaka, Japan). Mouse dendritic cells (DC2.4, Sigma-Aldrich) were cultured in RPMI 1640 medium, GlutaMAX supplement, 
HEPES (Gibco, Carlsbad, CA, USA) supplemented with 10\% heat-inactivated FBS, and 1\% $(v / v)$ NEAA. The cells were maintained at $37^{\circ} \mathrm{C}$ in a humidified incubator with $5 \% \mathrm{CO}_{2}$.

\subsection{Preparation of Mouse Bone Marrow-Derived Macrophage Cells (BMDMs)}

Seven-week-old female C57BL/ 6 mice (Charles River Laboratories Japan, Yokohama, Japan) were housed in a pathogen-free environment. All protocols were approved by the Animal Care and Use Committee of the National Institute for Materials Science (64-2020-5). To collect the BM cells, the mice were euthanized by intraperitoneal injection of an overdose of medetomidine-midazolam-butorphanol. Primary cells were harvested from the tibiae and femora of the female C57BL/ 6 mice. Red blood cells were removed from the BM using lysing buffer containing $155 \mathrm{mM} \mathrm{NH}_{4} \mathrm{Cl}, 10 \mathrm{mM} \mathrm{KHCO}_{3}$, and $0.1 \mathrm{mM}$ EDTA. To induce BMDMs, $5 \times 10^{5} \mathrm{BM}$ cells were plated in growth medium (RPMI 1640 medium supplemented with GlutaMAX supplement, HEPES, 10\% heat-inactivated FBS, 1\% penicillinstreptomycin (P/S) (100X, Thermo Fisher Scientific), and 1\% ( $v / v)$ NEAA) for 4 h to remove adherent cells. The supernatant was centrifuged at $300 \times g$ for $5 \mathrm{~min}$ at $4{ }^{\circ} \mathrm{C}$. The collected cells were cultured in growth medium supplemented with $55 \mu \mathrm{M} 2$-mercaptoethanol (Gibco, Carlsbad, CA, USA) and $100 \mathrm{ng} / \mathrm{mL}$ recombinant murine macrophage colony stimulating factor (Peprotech, Cranbury, NJ, USA) for 13 days.

\subsection{Immunostimulatory Assay}

After three or four passages, RAW264 cells or DC2.4 cells were suspended in media supplemented with $1 \% \mathrm{P} / \mathrm{S}$ and seeded into a 96-well plate at a density of $1 \times 10^{5}$ cells $/$ well $\left(5.59 \times 10^{5}\right.$ cells $\left./ \mathrm{mL}, 180 \mu \mathrm{L}\right)$. BMDMs were seeded in a 96-well plate at a density of $0.56 \times 10^{5}$ cells $/$ well $\left(3.15 \times 10^{5}\right.$ cells $\left./ \mathrm{mL}, 180 \mu \mathrm{L}\right)$. After incubation for $18 \mathrm{~h}, 20 \mu \mathrm{L}$ of $40 \mu \mathrm{M}$ ODNs was added to the culture medium to a final concentration of $2 \mu \mathrm{M}$. The cells were then incubated for $24 \mathrm{~h}$, and the supernatants and cells were collected. The relative transcript levels of interleukin (IL)-6, IL-12, and interferon (IFN)- $\beta$ were evaluated by reverse transcription/real-time quantitative polymerase chain reaction (RT/RQ-PCR), following previously reported protocols, and normalized to that of glyceraldehyde-3phosphate dehydrogenase (GAPDH) as housekeeping gene [9].

The concentration of murine interleukin (mIL)- 6 secreted into the culture medium was measured using the mouse IL-6 ELISA Ready-Set-Go kit (Thermo Fisher Scientific) according to the manufacturer's instructions.

\subsection{Statistical Analysis}

The data were statistically evaluated via one-way analysis of variance, followed by Tukey's multiple range test for comparison between test groups and Dunnett's multiple comparison test for comparison with the control group. All statistical analyses were performed using GraphPad Prism version 8.2.0.

\section{Results}

\subsection{Effect of CpG Motif Position in Anti-Parallel Duplex/G4 ODNs on G4 Formation and Immunostimulant Function}

All CpG ODN sequences evaluated in this study are listed in Table 1 . To evaluate the effect of the number and position of CpG motifs on topology and cytokine production, we designed a new monomeric G4 CpG ODN with a CpG motif added to each functional loop in the duplex/G-quadruplex-based aptamer RE31, a structurally analyzed thrombinbinding aptamer [15-17]. RE31 consists of a duplex region with six pairs of nucleotides and a quadruplex region with two G-tetrads and three functional loops, hereafter called G0.0.0 (Figure 1). Based on the $5^{\prime}$ to $3^{\prime}$ positions, the molecule is named G4 (G), followed by the number of CpG motifs (GTCGTT) in the first, second, and third functional loops. The CpG motif modification in the loop is labeled as 0,1 , or 2, according to the number of $\mathrm{CpG}$ motifs representing the separation between the loops. 


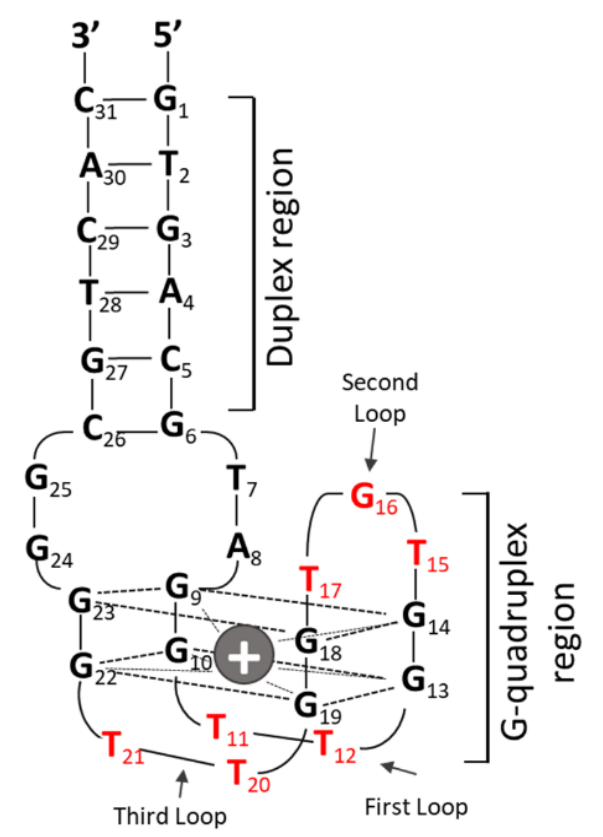

Figure 1. Structure of RE31 used as a scaffold to improve immunostimulatory function of CpG ODNs. The schematic represents the G0.0.0 design as the basic structure used in this study. The two G-tetrads in G0.0.0 are separated by three loops (L). Red colors in the G0.0.0 structures indicate loop sequences, where $T_{11}$ and $T_{12}$ form the first loop, $T_{15}, G_{16}$ and $T_{17}$ form the second loop, and $T_{20}$ and $\mathrm{T}_{21}$ form the third loop. The loops were modified by insertion of CpG motifs.

To investigate whether the position of the CpG motif in different functional loops affects the topology, we first synthesized three modified G0.0.0s containing two CpG motifs in each functional loop: G2.0.0, with two CpG motifs in the first loop, G0.2.0, with two CpG motifs in the second or central loop, and G0.0.2, with two CpG motifs in the third loop. The CD spectra of the designed G4-based CpG ODNs are shown in Figure 2. The $\mathrm{CD}$ measurement of G0.0.0 at $25^{\circ} \mathrm{C}$ (black line) showed two positive peaks at $247 \mathrm{~nm}$ and $294 \mathrm{~nm}$, with one negative peak at $265 \mathrm{~nm}$ (Figure 2a), which is characteristic of an antiparallel topology. CD measurement of CpG ODNs to study the effect of functional loop modifications showed that two CpG motifs in the first (G2.0.0) or third (G0.0.2) loops did not affect the antiparallel topology, which is seen in G0.0.0. However, when two CpG motifs were introduced into the second loop (G0.2.0), a positive peak at $295 \mathrm{~nm}$ and a negative peak at $265 \mathrm{~nm}$ were observed with a stepwise shift to $282 \mathrm{~nm}$ and $260 \mathrm{~nm}$, respectively (Figure 2c). We assumed that the formation of G0.2.0 was not stable at $25^{\circ} \mathrm{C}$; therefore, we attempted to observe the topology at a lower temperature $\left(10^{\circ} \mathrm{C}\right)$. The $\mathrm{CD}$ spectrum of G0.2.0 at $10{ }^{\circ} \mathrm{C}$ did not show any features characteristic of antiparallel topology, similar to the spectrum at $25^{\circ} \mathrm{C}$. These results indicated that the insertion of the $\mathrm{CpG}$ motif in the first and third loops preserved the antiparallel topology, whereas CpG ODNs with additional CpG motifs in the second loop failed to maintain the antiparallel topology.

Next, we evaluated the immunostimulatory activity of G4-based CpG ODNs by examining mRNA expression in mouse macrophage-like RAW264 cells, since the transcript levels of inflammatory cytokine and type I interferon induced by CpG ODNs show the same tendency as protein secretion levels of these factors $[9,19,20]$. As shown in Figure 3, the position of the $\mathrm{CpG}$ motifs influenced cytokine production. G4 CpG ODNs having two CpG motifs in the first (G2.0.0) or second (G0.2.0) loops showed high IL-6, IL-12, and IFN- $\beta$ mRNA induction. Conversely, G4 CpG ODN with CpG motif in the third loop had a lower immunostimulatory effect. To investigate the effect of the number of $\mathrm{CpG}$ motifs in the first functional loop, we employed G1.0.0, which had only one CpG motif. As shown in Figure S1, increasing the number of CpG motifs in the first functional loop was responsible for cytokine enhancement. Furthermore, G2.0.0 showed a dose-dependent immunostimulatory effect on RAW264 cells (Figure S2). 
a

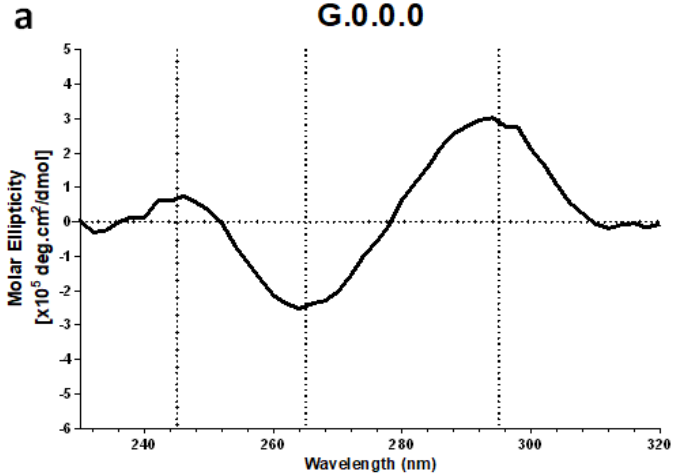

C

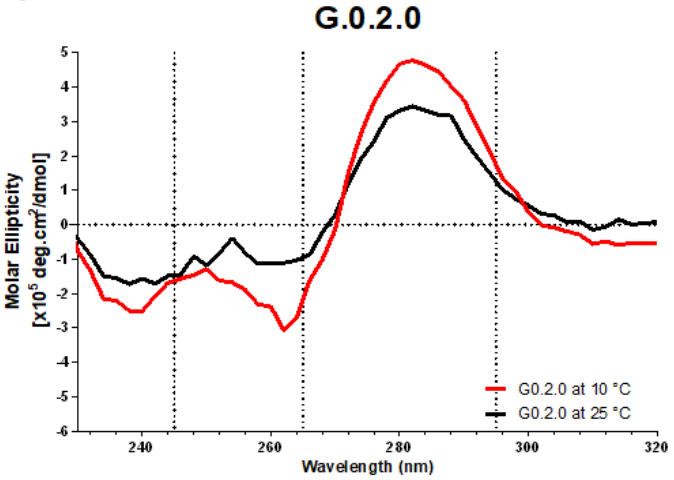

b

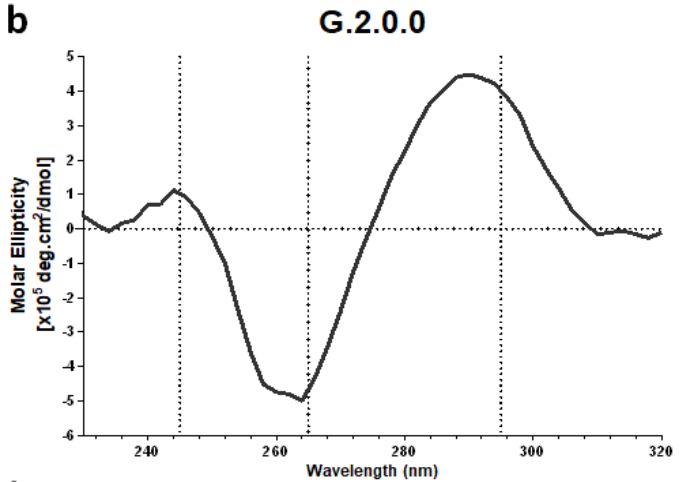

d

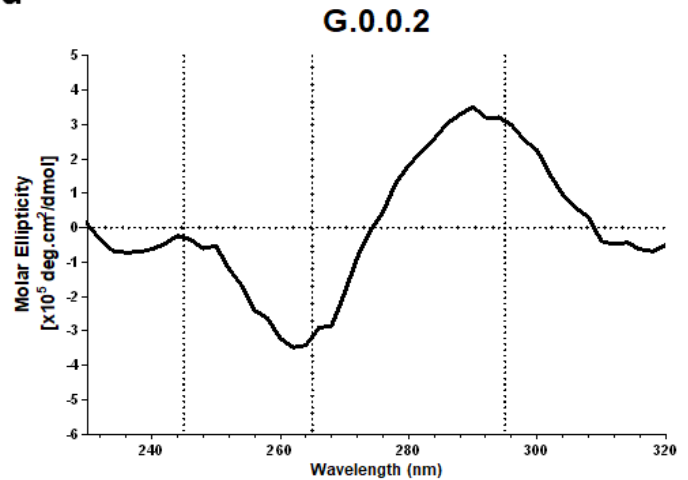

Figure 2. Circular dichroism spectra of (a) G0.0.0, (b) G2.0.0, (c) G0.2.0, and (d) G0.0.2 in D-PBS at $25^{\circ} \mathrm{C}$ (black line) or $10{ }^{\circ} \mathrm{C}$ (red line). CpG ODNs concentration is $2 \mu \mathrm{M}$.
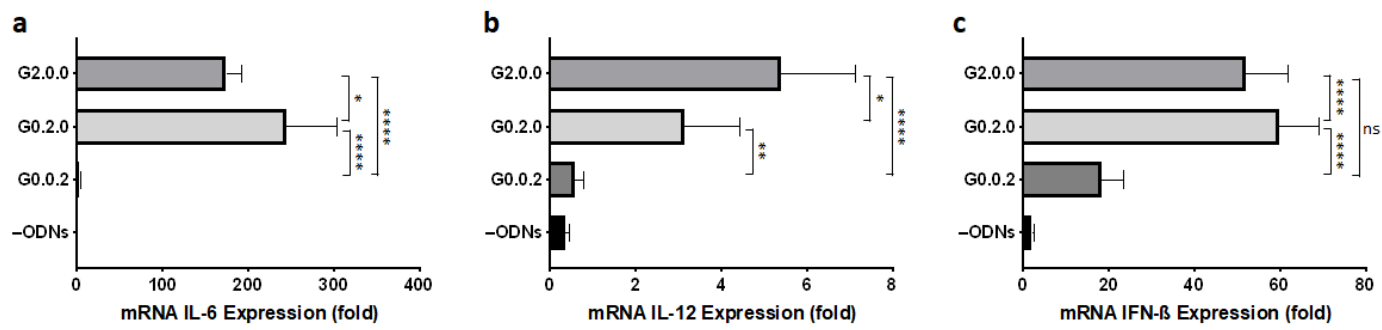

Figure 3. Induction of (a) IL-6, (b) IL-12, and (c) IFN- $\beta$ mRNA transcription in RAW264 cells $\left(1 \times 10^{5}\right.$ cells/well) stimulated with $2 \mu \mathrm{M}$ duplex/G-quadruplex-based CpG ODNs. G2.0.0, G0.2.0, and G0.0.2 contain two CpG motifs in first, second, and third loop, respectively. -ODNs indicate RAW264 cells with only D-PBS buffer added. Data are presented as the mean \pm SD $(n=5) .{ }^{* * *} p<0.0001,{ }^{* *} p<0.01,{ }^{*} p<0.05$, ns: not significantly different (one-way analysis of variance, Tukey's multiple comparisons test for comparison with other groups).

These results suggest that the CpG motif in the first loop in antiparallel type duplex/G4 maintains an antiparallel topology and can also induce the highest cytokine mRNA levels. We further investigated the enhancement of the immunostimulatory effect using G2.0.0.

\subsection{Influence of Nucleotide Connectors between G-Tract and CpG Loop on G4 Formation and Immunostimulatory Activity}

To improve the immunostimulatory effect of antiparallel type G4 CpG ODNs, we designed a variant of G2.0.0 with additional nucleotide connectors between G-tetrad and the CpG loop. Gc2c.0.0, in which cytosine (C) was inserted into the $5^{\prime}$ and $3^{\prime}$ ends of the CpG motif, showed an antiparallel topology with two positive peaks at $245 \mathrm{~nm}$ and $293 \mathrm{~nm}$ and a negative peak at $262 \mathrm{~nm}$ in CD spectroscopy at $25^{\circ} \mathrm{C}$ (Figure 4a). The other three variants, Ga2a.0.0, Ga2c.0.0, and Gc2a.0.0, showed a shift in the positive peak at $295 \mathrm{~nm}$ to lower wavelengths, and the negative peak at $265 \mathrm{~nm}$ disappeared. However, upon CD spectrum measurement at $10^{\circ} \mathrm{C}, \mathrm{Ga2a} .0 .0$ and Ga2c.0.0 showed the same $\mathrm{CD}$ spectrum as 
that of G0.0.0, with two positive peaks at around $245 \mathrm{~nm}$ and $295 \mathrm{~nm}$ and one negative peak around $265 \mathrm{~nm}$. However, Gc2a.0.0 did not show a typical CD spectrum characteristic of antiparallel topology even at $10^{\circ} \mathrm{C}$. We assumed that the insertion of nucleotides between the G-tetrad and the loop reduces the stability of G4 formation; however, cytosine insertion in both the $3^{\prime}$ and $5^{\prime}$ termini of the CpG motif had no effect on the G4 structure.

a

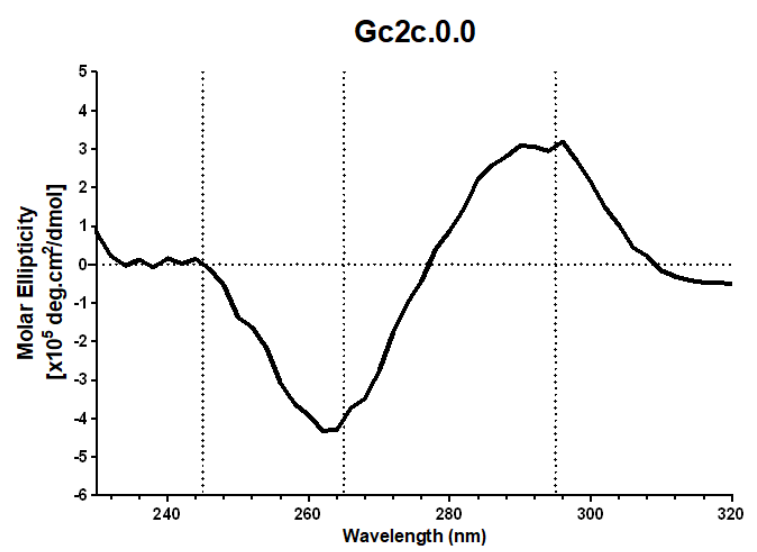

C
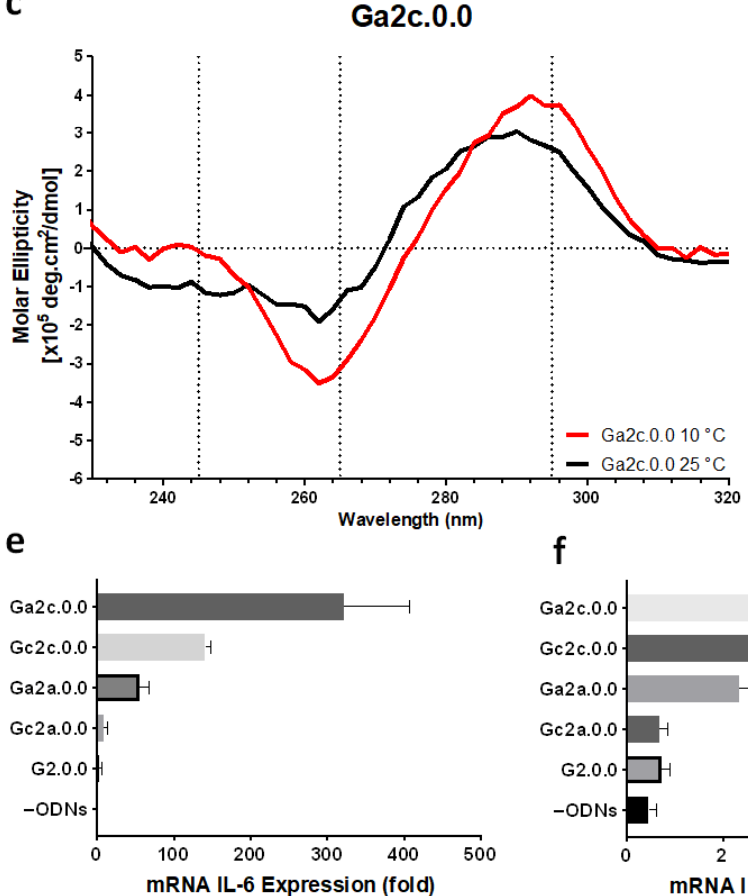

b

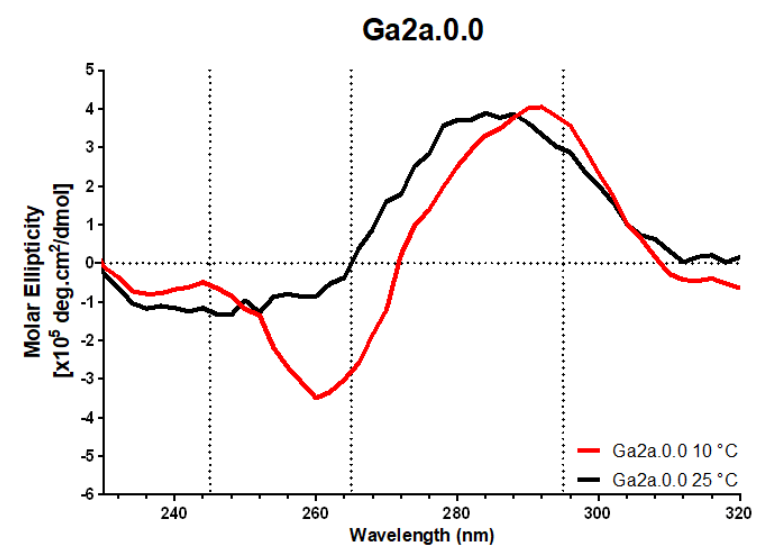

d

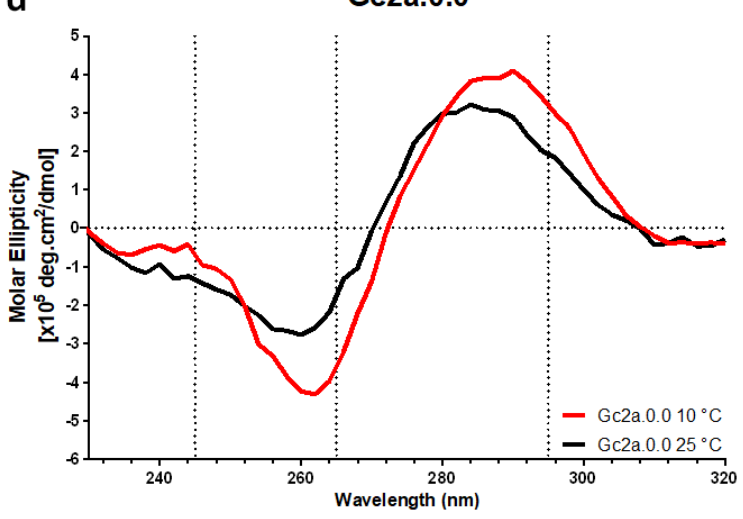

g

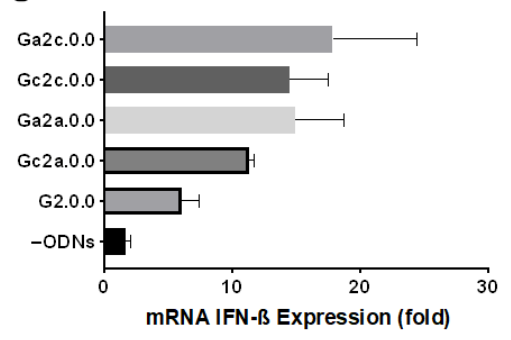

Figure 4. Effect of flanking sequence between the CpG motif and G-tetrad in the first loop on antiparallel G-quadruplex formation and cytokine induction. Circular dichroism spectra of (a) Gc2c.0.0, (b) Ga2a.0.0, (c) Ga2c.0.0, and (d) Gc2a.0.0 at $25{ }^{\circ} \mathrm{C}$ (black line) or $10{ }^{\circ} \mathrm{C}$ (red line) were observed. Relative expression level of (e) IL-6, (f) IL-12, and (g) IFN- $\beta$ mRNA transcription in RAW264 cells stimulated by G2.0.0, Ga2a.0.0, Gc2c.0.0, Ga2c.0.0, and Gc2a.0.0. -ODNs indicate RAW264 cells with only D-PBS buffer added. Data are presented as the mean $\pm \operatorname{SD}(n=5)$.

The immunostimulatory effects of these variants were evaluated. As shown in Figure $4 \mathrm{e}-\mathrm{g}$, an additional connector between the G-tetrad and the CpG motif could induce cytokine at levels higher than those obtained using G2.0.0 containing only two CpG motifs. In particular, the two variants with cytosine at the $3^{\prime}$-end of the CpG motif, Ga2c.0.0 and Gc2c.0.0, showed high cytokine induction ability.

Next, we examined variants in which two bases were inserted between the G-tract and the CpG motif. Figure 5 shows the CD spectra and cytokine production of G4 CpG 
ODN variants with two-base insertions. We designed four variants: Gac2ac.0.0, Gca2ac.0.0, Gca2ca.0.0, and Gac2ca.0.0. Only Gac2ac.0.0 showed typical CD spectra characteristics of antiparallel topology when measured at $25{ }^{\circ} \mathrm{C}$. In the CD spectra of the other three variants, the positive peak at $295 \mathrm{~nm}$ was shifted to around $280-285 \mathrm{~nm}$, and the negative peak at $265 \mathrm{~nm}$ was shifted to around $260 \mathrm{~nm}$. The shifted peaks of Gca2ac.0.0, Gca2ca.0.0, and Gac2ca.0.0 did not revert to the typical antiparallel topology wavelengths even when measured at $10^{\circ} \mathrm{C}$. Moreover, the addition of three bases between the G-tract and CpG motif destabilized the antiparallel topology (Table S1, Figure S3). The immunostimulatory activity of the two-base inserted variant was compared with that of the linear CpG ODN with two CpG motifs, G2.0.0, and single-base inserted variants. All nucleotide-inserted modifications showed a higher immunostimulatory effect than the linear CpG ODN and G2.0.0. The one-base inserted variant induced a much stronger immune response than the two-base inserted variant. This indicated that the optimized spacer length between G-tracts and CpG motifs increased the immunostimulatory efficiency of antiparallel G4 CpG ODNs.

a
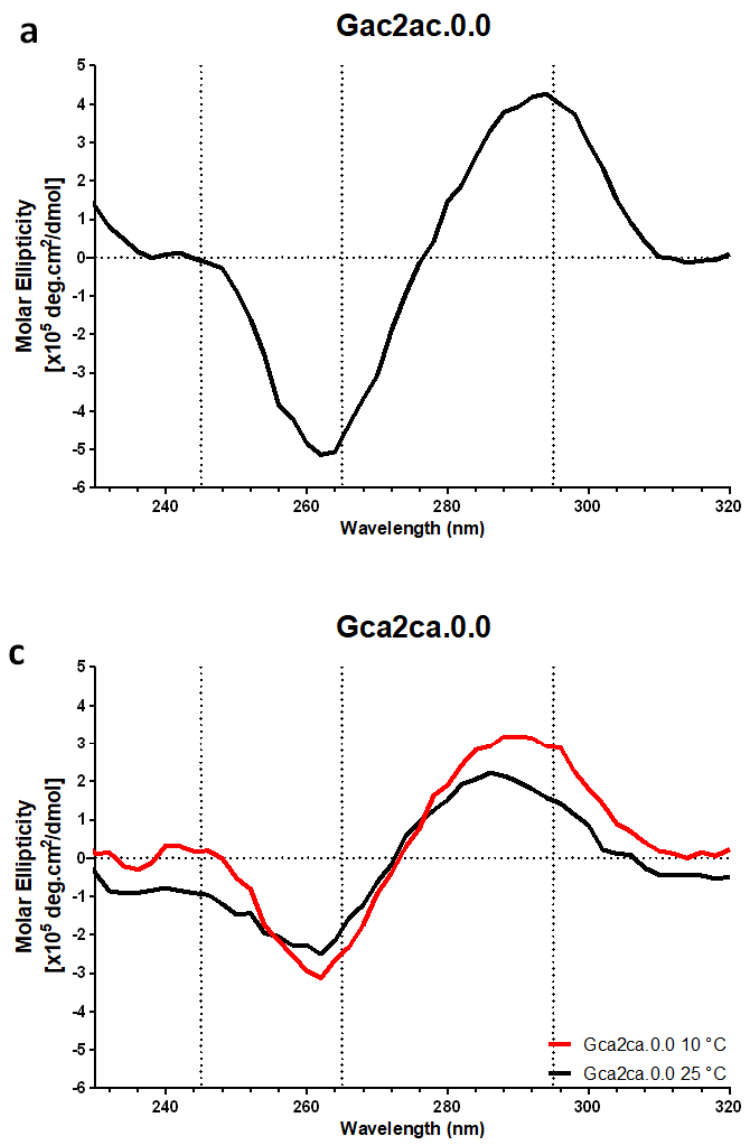

e

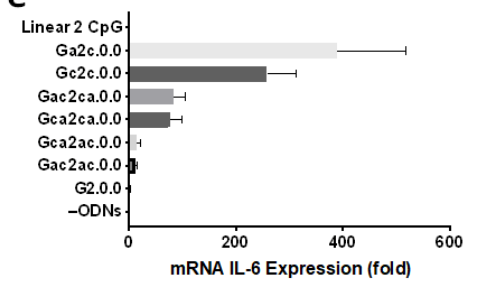

b
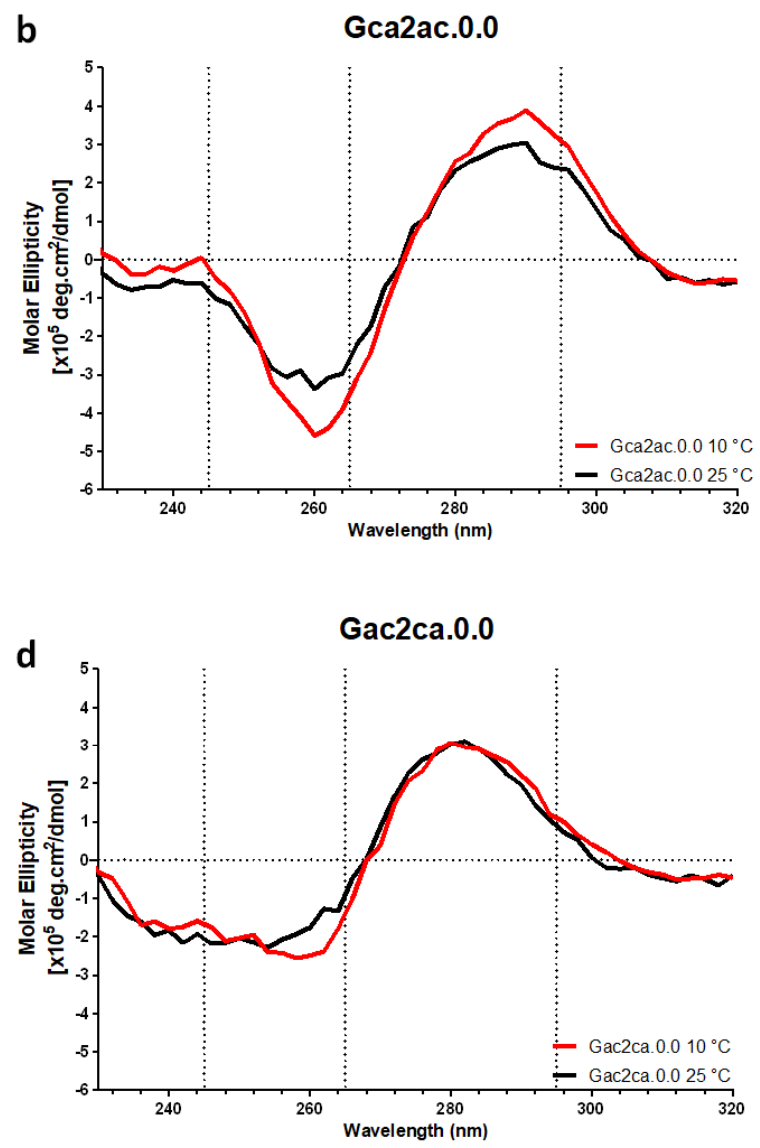

g

Figure 5. Effect of flanking sequence between the CpG motif and G-tetrad in the first loop on antiparallel G-quadruplex formation and cytokine induction. Circular dichroism spectra of (a) Gac2ac.0.0, (b) Gca2ac.0.0, (c) Gca2ca.0.0, and (d) Gac2ca.0.0 at $25{ }^{\circ} \mathrm{C}$ (black line) or $10^{\circ} \mathrm{C}$ (red line) were observed. Relative expression level of (e) IL-6, (f) IL-12, and (g) IFN-ß mRNA transcription in RAW264 cells stimulated by Linear 2CpG, G2.0.0, Ga2a.0.0, Gc2c.0.0, Gac2ac.0.0, Gac2ca.0.0, Gca2ac.0.0, and Gca2ca.0.0. -ODNs indicate RAW264 cells with only D-PBS buffer added. Data are presented as the mean \pm SD $(n=5)$. 


\subsection{Electrophoresis Analysis of Anti-Parallel G4 CpG ODNs}

Next, we analyzed the structure of G4 CpG ODNs by PAGE. As shown in Figure 6, we compared the mobility of our designed CpG ODNs with that of linear ODNs with the same nucleotide base length. All G4 CpG ODNs migrated faster than the linear ODNs in both the presence and absence of potassium ions in the annealing condition. These results suggest that G4 CpG ODN forms a stable, compact structure that is independent of the presence of potassium ions.
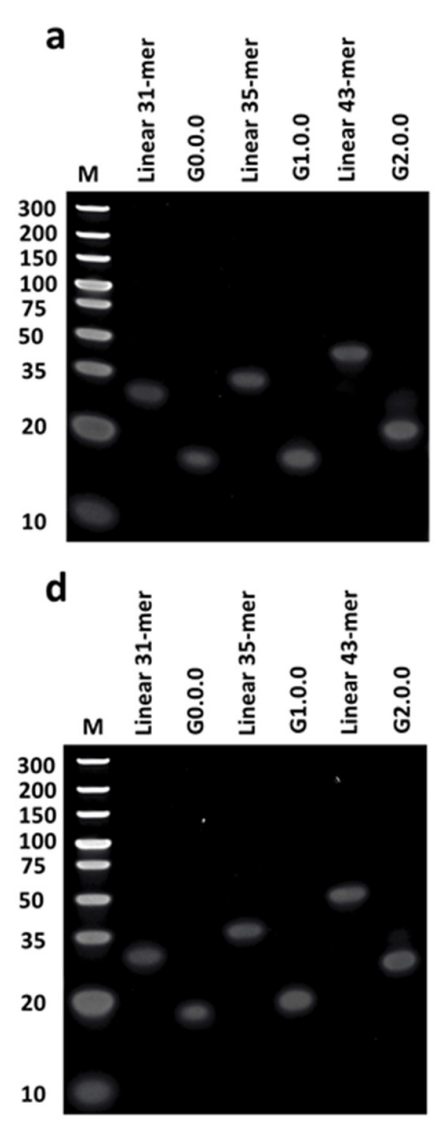

b
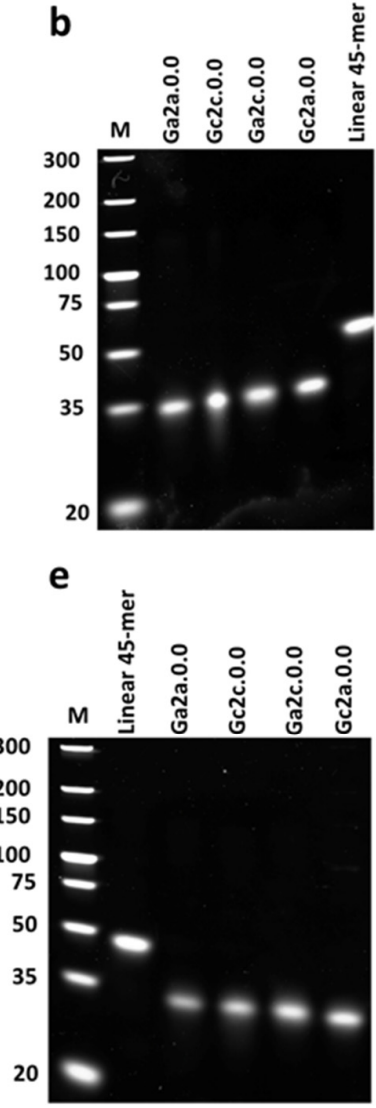

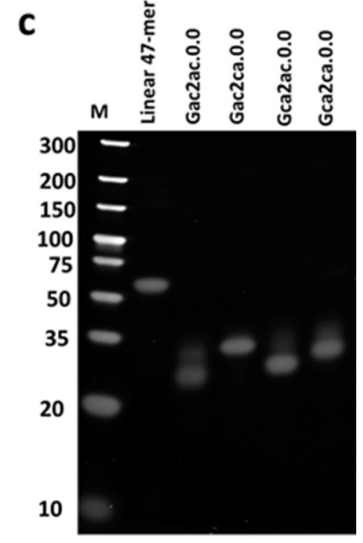

f

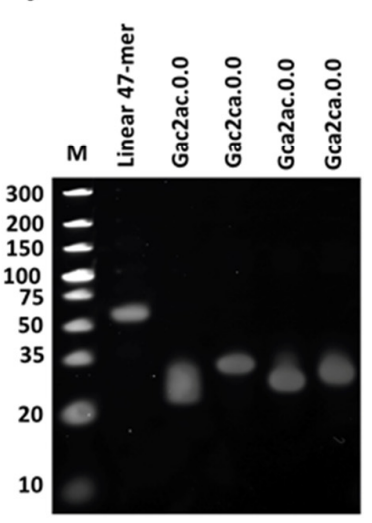

Figure 6. Polyacrylamide gel electrophoresis of G-quadruplex CpG ODNs. Samples were reconstructed in (a-c) D-PBS buffer (containing $4 \mathrm{mM} \mathrm{K}^{+} 150 \mathrm{mM} \mathrm{Na}^{+}$) and (d-f) Tris-HCl buffer by heating at $95{ }^{\circ} \mathrm{C}$ for $5 \mathrm{~min}$ followed by gradual cooling to $30{ }^{\circ} \mathrm{C}$ within $30 \mathrm{~min}$, followed by cooling to $4{ }^{\circ} \mathrm{C}$. Electrophoresis was performed using a $12 \%$ polyacrylamide gel in $0.5 \times$ Tris-Borate-EDTA buffer supplemented by $4 \mathrm{mM} \mathrm{KCl}$. M is a 10-bp DNA marker.

\subsection{Serum and Thermal Stability of G4 Cp G ODNs}

To evaluate the stability of antiparallel G4 CpG ODNs against nucleases in serum, ODNs were incubated in $20 \%(v / v)$ FBS at $37^{\circ} \mathrm{C}$ for $0 \mathrm{~h}, 1 \mathrm{~h}, 2 \mathrm{~h}, 4 \mathrm{~h}$, and $24 \mathrm{~h}$ and then subjected to electrophoresis (Figure 7). The results showed that the linear 2 CpG ODN was $75 \%$ and $90 \%$ degraded after 1-h and 4-h incubation, respectively. All G4 CpG ODNs degraded more slowly than the linear 2 CpG ODNs in serum. This result indicates that stability of ODNs in serum can be acquired by forming a G4 structure. In addition, the length of the inserted CpG sequence in the first external loop did not affect serum stability up to four bases.

To perform the thermodynamics analysis of designed G4 CpG ODNs, we used the CD melting temperature analysis from the unfolded formation $\left(5^{\circ} \mathrm{C}\right)$ to the folded formation $\left(90^{\circ} \mathrm{C}\right.$ ) (Figure S4). The melting temperatures of $\mathrm{CpG}$ ODNs were determined from the CD melting curves at $295 \mathrm{~nm}$, as shown in Table 2. The Tm value of G0.0.0 without CpG was approximately $42{ }^{\circ} \mathrm{C}$, whereas the Tm value of the CpG motif-inserted G4 CpG ODN 
decreased to below $35^{\circ} \mathrm{C}$. This indicated that the insertion of long sequences in the loop region induced thermal denaturation of the antiparallel G4 structure.

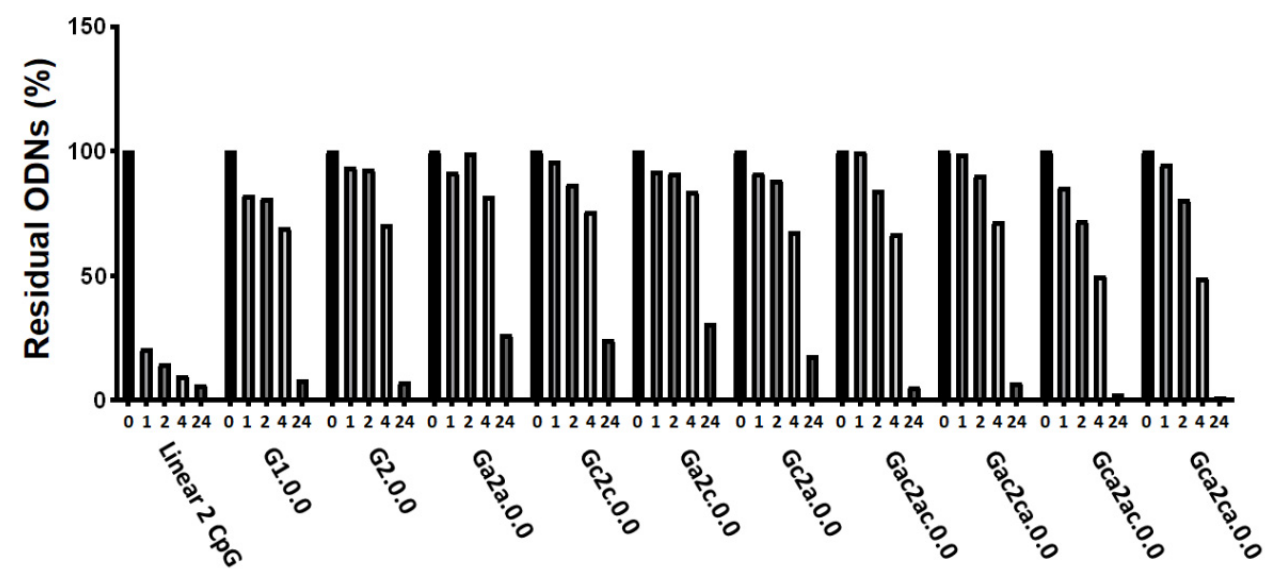

Figure 7. Degradation of G-quadruplex CpG ODNs in serum. Each ODN was incubated in $20 \%$ fetal bovine serum for $1,2,4$, and $24 \mathrm{~h}$ at $37^{\circ} \mathrm{C}$. Percentage of residual ODN was analyzed by polyacrylamide gel electrophoresis with SYBR Gold staining.

Table 2. Melting temperature at the first cooling and second heating of antiparallel ODNs with CpG motif substitutions in the first functional loop, with or without additional connectors, obtained from CD-melting curves in D-PBS.

\begin{tabular}{|c|c|c|c|c|c|}
\hline \multirow{2}{*}{ Name } & \multicolumn{3}{|c|}{ Loop Sequence } & \multirow{2}{*}{$\begin{array}{c}\text { Tm at 1st } \\
\text { Cooling }\left({ }^{\circ} \mathrm{C}\right)\end{array}$} & \multirow{2}{*}{$\begin{array}{c}\text { Tm at } 2 \mathrm{nd} \\
\text { Heating }\left({ }^{\circ} \mathrm{C}\right)\end{array}$} \\
\hline & 1st & 2nd & 3rd & & \\
\hline G0.0.0 & $\mathrm{TT}$ & TGT & $\mathrm{TT}$ & 41.8 & 42.8 \\
\hline G1.0.0 & GTCGTT & TGT & $\mathrm{TT}$ & 40.2 & 41.2 \\
\hline G2.0.0 & GTCGTTTTGTCGTT & TGT & $\mathrm{TT}$ & 35.6 & 35.6 \\
\hline G0.0.2 & $\overline{\mathrm{TT}} \overline{ } \overline{ }$ & TGT & GTCGTTTTGTCGTT & 32.9 & 33.4 \\
\hline Ga2a.0.0 & AGTCGTTTTGTCGTTA & TGT & $\overline{\mathrm{TT}} \overline{ }$ & 33.6 & 34.9 \\
\hline Gc2c.0.0 & CETCGTTTTGTCGTTC & TGT & $\mathrm{TT}$ & 32.5 & 32.8 \\
\hline Ga2c.0.0 & AGTCGTTTTGTCGTTC & TGT & TT & 30.2 & 29.5 \\
\hline Gc2a.0.0 & CETCGTTTTGTCGTTA & TGT & $\mathrm{TT}$ & 25.9 & 25.2 \\
\hline Gac2ac.0.0 & ACGTCGTTTTGTCGTTAC & TGT & $\mathrm{TT}$ & 33.6 & 34.6 \\
\hline Gca2ac. 0.0 & CAGTCGTTTTETCGTTAC & TGT & $\mathrm{TT}$ & 32.5 & 34.7 \\
\hline Gca2ca.0.0 & CAGTCGTTTTGTCGTTCA & TGT & $\mathrm{TT}$ & 34.9 & 30.3 \\
\hline
\end{tabular}

Note: CpG motifs are underlined. All ODNs have a phosphodiester backbone.

\subsection{Immune Responses of Mouse Dendritic Cells and BMDMs}

We assessed the immunostimulatory potential of antiparallel G4 CpG ODNs in mouse dendritic cells and primary macrophages. In this investigation, we employed G2.0.0, Gc2c.0.0, and Ga2c.0.0, as well as the linear 2 CpG motif. As shown in Figure 8, Gc2c.0.0 and Ga2c.0.0, antiparallel type G4 CpG ODNs with one cytosine in the $3^{\prime}$ flanking the sequence of $\mathrm{CPG}$ motifs, showed high immunostimulatory cytokine induction in DC2.4 dendritic cells and BMDMs, similar to the results obtained in RAW264 cells. We also examined the IL-6 protein production level in RAW264 cells and BMDMs induced by G4 CpG ODNs (Figure 9). IL-6 protein levels induced by Gc2c.0.0 and Ga2c.0.0 were significantly higher than those induced by G2.0.0, whereas linear 2 CpG did not induce IL-6. These results confirmed the positive correlation between the transcription level and the secretion level of IL-6. The results also showed that antiparallel G4 CpG ODNs can activate primary immune cells, not only cell lines. 

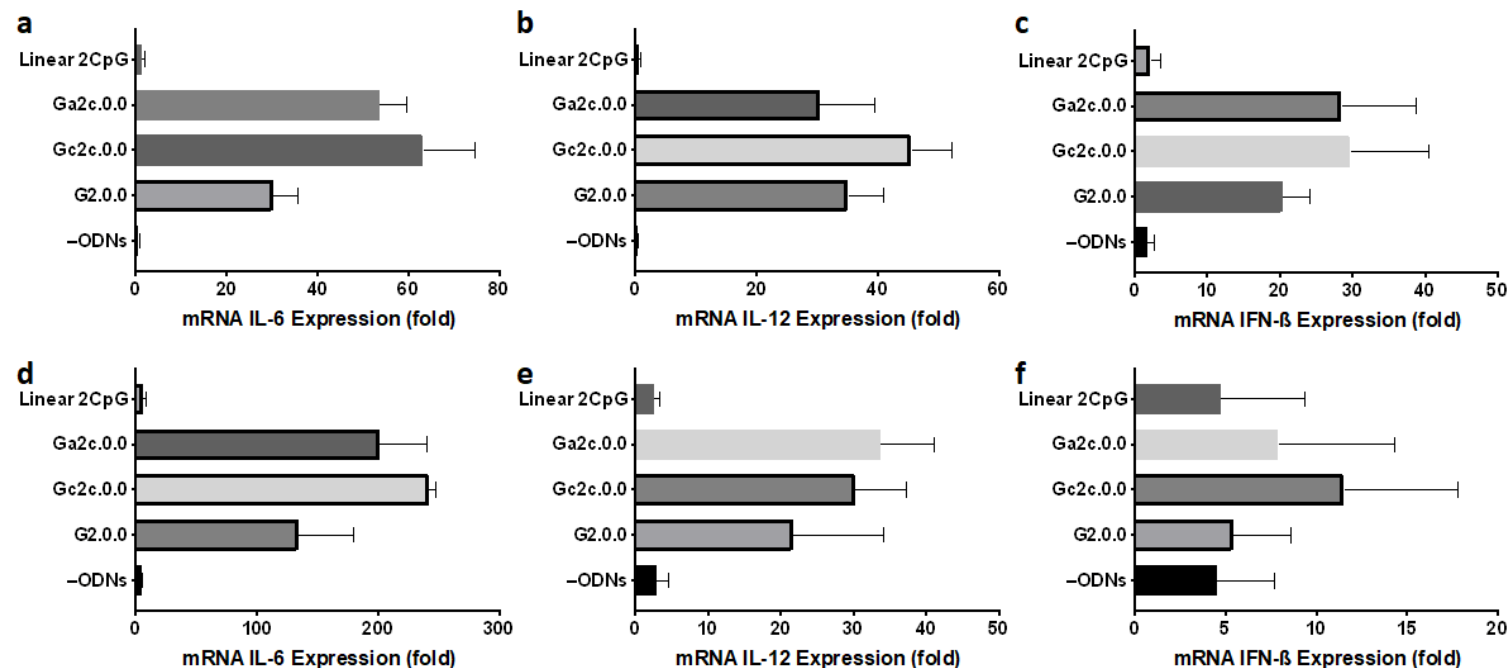

Figure 8. Immunostimulatory effect of antiparallel type CpG ODNs in (a-c) DC2.4 mouse dendritic cells and (d-f) bone marrow-derived macrophage cells (BMDMs). Messenger RNA expression levels of cytokines (a,d) IL-6, (b,e) IL-12, and $(\mathbf{c}, \mathbf{f})$ IFN- $\beta$ were determined using RT/RQ-PCR. The cells (DC2.4; $1 \times 10^{5}$ cells/well, BMDMs; $0.56 \times 10^{5}$ cells/well) were stimulated by $2 \mu \mathrm{M}$ G2.0.0, Gc2c.0.0, and Ga2c.0.0 and linear 2CpG for 24 h. -ODNs indicate RAW264 cells with only D-PBS buffer added. Data are presented as mean $\pm \mathrm{SD}(n=5)$.
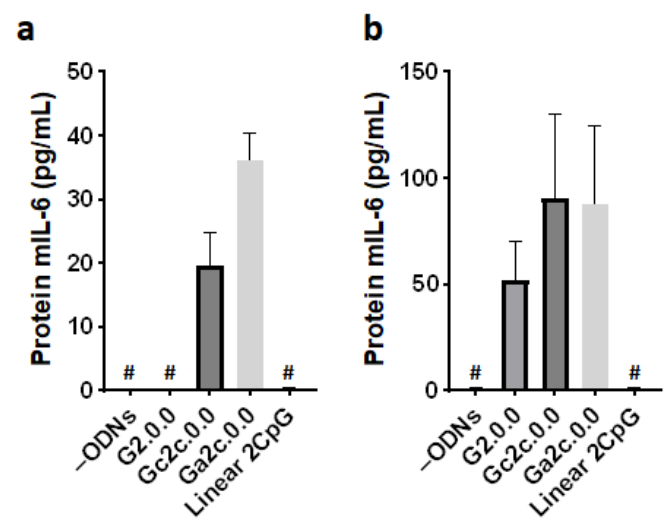

Figure 9. The level of IL-6 secreted into the culture medium induced by antiparallel type CpG ODNs. (a) RAW264 cells and (b) bone marrow-derived macrophage cells (BMDMs). The cells (RAW264; $1 \times 10^{5}$ cells/well, BMDMs; $0.56 \times 10^{5}$ cells/well) were stimulated by $2 \mu \mathrm{M} \mathrm{G2.0.0,} \mathrm{Gc2c.0.0,} \mathrm{Ga2c.0.0,}$ and linear 2CpG for $24 \mathrm{~h}$, the supernatants were collected, and the secretion levels of interleukin-6 in medium were determined by ELISA. -ODNs indicate RAW264 cells with only D-PBS buffer added. Data are presented as mean $\pm \mathrm{SD}(n=5)$. The hash tag (\#) indicates the lower detection limit $(7.8 \mathrm{pg} / \mathrm{mL})$.

\section{Discussion}

Recently, G4 structures have been found to increase nuclease tolerance in serum and enhance the immunostimulatory activity of CpG ODNs both in vitro and in vivo [9,10]. The formation of G4 structures has three possible topologies: parallel, antiparallel, and hybrid. The topology of G-quadruplex depends on loop lengths and sequences [9] and the type and concentration of cations [21,22]. In our study, we developed an intramolecular antiparallel G4 CpG ODN by inserting CpG motifs into functional loops of G4 ODNs. In the construction of new G4 CpG ODNs, G4 forming an antiparallel topology was selected by CD analysis, followed by the measurement of immunostimulatory activity. The results showed that by inserting the CpG motif into the first external loop of antiparallel G4, G4 CpG ODNs that maintain an antiparallel structure and exhibit a high immunostimulatory function can be obtained. Furthermore, we found that the additional single nucleotide connector between the G-tetrad and CpG motif as a flanking sequence improved the induction of cytokines in immune cells, including primary immune cells differentiated from mouse BMs. 
In this study, we used the thrombin-inhibiting DNA aptamer, RE31, as a scaffold for preparing antiparallel G4 CpG ODNs. The position of the CpG motif in the G4 structure influenced the conformation of G4 CpG ODNs. G4 CpG ODNs with CpG motifs in the first and third loops maintained the antiparallel topology, whereas G4 CpG ODNs with CpG motifs in the second loop changed the topology to a random structure. Krauss et al. (2016) reported that the first and third loops of RE31 have important interactions with thrombin, and the second loop plays a crucial role in G-quadruplex formation [17]. The second loop (TGT) is involved in the transition from a linear structure to a G-quadruplex structure by linking through a reverse Hoogsteen hydrogen bond network [17]. Thus, in RE31, the first and third loops can be considered insertion sites for the $\mathrm{CpG}$ motifs that maintain the antiparallel G4 structure. The position of the CpG motif in the G4 structure also influences the immunostimulatory activity of G4 CpG ODNs. CpG ODNs are taken up by immune cells by endocytosis and bind to TLR9 in the endosomes/lysosomes compartment, inducing cytokinesis. The production level of marker proteins for immune activity, such as IL-6, IL-12, and IFN- $\beta$, depends on the interaction between CpG ODNs and TLR9. As shown in Figure 1, there are four guanine linkages $\left(\mathrm{G}_{22}-\mathrm{G}_{25}\right)$ on the $3^{\prime}$-terminal side of the third loop of RE31, suggesting that cytosine and guanine of the CpG motif inserted into the third loop may interact with these polyguanines. This may have resulted in weaker interaction of the CpG motif in the third loop with TLR9, resulting in lower cytokine induction. To clarify this, further structural analysis of G4 CpG ODNs by NMR and X-ray structure analysis, as well as analysis of the interaction between TLR9 and CpG ODNs by immunoprecipitation and surface plasmon analysis, will be necessary.

We previously reported that spaces between the CpG motifs and G-tracts increased the stimulation efficiency of hybrid G4 CpG ODNs in RAW264 cells [9]. Based on our previous results, we added nucleotides between the G-tetrad and CpG motifs to enhance cytokine expression and observed the stability of the structure. We modified the length of the first functional loop by adding connectors between the CpG motifs and G-tetrad as follows: one space with $\mathrm{A}$ or $\mathrm{C}$ at the first position (between the G-tetrad and $\mathrm{CpG}$ motif in the $5^{\prime}$ to $3^{\prime}$ direction) and second position (between the CpG motif and G-tetrad in the $5^{\prime}$ to $3^{\prime}$ direction). We prepared eight variants of the antiparallel G4 CpG ODN, G2.0.0, of which only Gc2c.0.0 and Gac2ac.0.0 maintained their antiparallel topology at $25^{\circ} \mathrm{C}$. Other sequences, such as Ga2a.0.0, Ga2c.0.0, Gca2ac.0.0, and Gca2ca.0.0, showed $\mathrm{CD}$ spectra characteristic of antiparallel topology when the temperature was decreased to $10^{\circ} \mathrm{C}$, whereas the remaining variants showed CD spectra characteristic of non-antiparallel structures even at low temperatures. We evaluated the immunostimulatory effects of these variants on immune cells. The variants that maintained the antiparallel G4 topology induced high levels of IL-6, IL-12, and IFN- $\beta$ mRNA transcription in RAW cells. These results indicated that stable retention of the antiparallel G4 structure resulted in high immunostimulatory activity.

Here, we stimulated dendritic cells and macrophages with our monomeric G4 CpG ODNs to observe their ability to produce cytokines that lead to signal communication between immune and non-immune cells [23-25]. These cytokines are responsible for the catabolic process called autophagy [26]. In RAW264 cells, Ga2c.0.0 and Gc2c.0.0 induced the highest mRNA and protein expression of mIL- 6 compared to other designs, as determined using RT/RQ-PCR and ELISA, respectively. We also successfully stimulated mouse macrophage primary cells with monomeric G4 CpG ODNs. Macrophages mediate interactions with dendritic cells [27]; therefore, we stimulated BMDMs with monomeric ODNs and found that these cells highly expressed cytokines both at the mRNA transcription of IL-6, IL-12, and IFN- $\beta$ and immunostimulatory IL- 6 production levels. The ability of Ga2c.0.0 and Gc2c.0.0 to stimulate both cell lines and primary cells suggests their potential for further application in vivo. Given the evidence, our new antiparallel G4 has a robust scaffold function to maintain antiparallel G4 CpG ODNs and increases the nucleases resistance and high cytokine level in primary and line cells. However, in vivo studies are needed to demonstrate its application as a vaccine adjuvant. 


\section{Conclusions}

We developed and characterized antiparallel duplex/G4-based CpG ODNs, which allowed us to understand the roles of each functional loop in the structure. We found that the distance between the G-tetrad and CpG motif is important to impart flexibility to the CpG motif that is necessary to be recognized by TLR9; however, the longest loop could not ensure the highest cytokine expression. Furthermore, one spacer was sufficient to stimulate and enhance cytokine production in both cell lines and primary cells. Our study provides useful information on G4 CpG ODNs for further in vivo applications.

Supplementary Materials: The following are available online at https:/ /www.mdpi.com/article/10 $.3390 /$ biom $11111617 / \mathrm{s} 1$, Table S1: G4 CpG ODNs having three flanking sequences on the $5^{\prime}$ and $3^{\prime}$ termini of $\mathrm{CpG}$ motifs. Figure S1: Effect of the number of $\mathrm{CpG}$ motifs on cytokine induction, Figure S2: Relative expression levels of (a) IL-6, (b) IL-12, and (c) IFN- $\beta$ in mouse macrophage-like RAW 264 cells stimulated with different concentrations of G2.0.0 $(0 \mu \mathrm{M}, 0.25 \mu \mathrm{M}, 0.5 \mu \mathrm{M}, 1 \mu \mathrm{M}, 2 \mu \mathrm{M}$, and $4 \mu \mathrm{M}$ ), Figure S3: Effect of three connectors between CpG number and G-tetrad at first loop on antiparallel G-quadruplex formation and cytokine induction, Figure S4: Melting temperature (Tm Value) analysis using CD-melting profile of $2 \mu \mathrm{M}$ of (a) G0.0.0, (b) G1.0.0, (c) G2.0.0, (d) G0.0.2, (e) Ga2a.0.0, (f) Gc2c.0.0, (g) Ga2c.0.0, (h) Gc2a.0.0, (i) Gac2ac.0.0, (j) Gca2ac.0.0, and (k) Gca2ca.0.0 in $1 \times$ D-PBS buffer (pH 7.4) in the presence of $4 \mathrm{mM} \mathrm{K}^{+}$and $150 \mathrm{mM} \mathrm{Na}^{+}$at $295 \mathrm{~nm}$.

Author Contributions: F.A.S. carried out the experiment, analyzed data, and wrote the first draft of the manuscript. A.T.T.T., K.H. and D.Z. helped to supervise the evaluation of the immunostimulatory effects of $\mathrm{CpG}$ ODN in cells. M.S. designed and carried out the animal experiment. A.B.W., S.H.S. and E.A.G.-R. helped supervise the project as well as the review and editing of the manuscript. T.Y. contributed to study conception, design, data interpretation, wrote the first draft of the manuscript, editing of manuscript. K.I. and K.T. supervised the G4 topology analysis. All authors have read and agreed to the published version of the manuscript.

Funding: This work was supported by the Japan Society for the Promotion of Science Kakenhi (grant numbers 18K04858 and 17KK0122). This research was supported by funding from International Cooperative Graduate School (ICGS) Fellowship under the "Institut Teknologi Bandung-NIMS Cooperative Graduate School Program".

Institutional Review Board Statement: The study was conducted according to the guidelines of National Institute Materials Sciences, and approved by the Animal Care and Use Committee of the National Institute for Materials Science (64-2020-5).

Informed Consent Statement: Not applicable.

Data Availability Statement: Not applicable.

Acknowledgments: The authors would like to acknowledge Xianglan Li, Shinya Hattori, and Satomi Kohara, as well as the other members of the NIMS Molecule and Material Synthesis platform for technical support. We are grateful to and appreciate Masayuki Takeuchi and Junko Aimi for their technical suggestions for the CD and UV analysis. We are thankful that this work was conducted at the NIMS Molecule and Material Synthesis platform, supported by the Nanotechnology Platform Program of the Ministry of Education, Culture, Sports, Science and Technology (MEXT), Japan.

Conflicts of Interest: The authors declare no conflict of interest.

\footnotetext{
Abbreviations

ODNs, oligodeoxynucleotides; CpG, cytosine-phosphate-guanine; G4, G-quadruplex; G4 CpG ODNs, G4-based CpG ODNs; IL-6, interleukin-6; IL-12, interleukin-12; IFN- $\beta$, interferon- $\beta$; TLR9, Toll-like receptor 9; PD, phosphodiester; DCs, dendritic cells; GAPDH, glyceraldehyde 3-phosphate dehydrogenase; ELISA, enzyme-linked immunosorbent assay; RT/RQ-PCR, reverse transcription/realtime quantitative-polymerase chain reaction; $\mathrm{CD}$, circular dichroism; UV, ultraviolet; TDS, thermal difference spectra; PAGE, polyacrylamide gel electrophoresis; BMDMs, bone marrow-derived macrophage cells.
} 


\section{References}

1. Kawasaki, T.; Kawai, T. Toll-like receptor signaling pathways. Front. Immunol. 2014, 5, 461. [CrossRef] [PubMed]

2. Shi, S.; Zhu, H.; Xia, X.; Liang, Z.; Ma, X.; Sun, B. Vaccine adjuvants: Understanding the structure and mechanism of adjuvanticity. Vaccine 2019, 37, 3167-3178. [CrossRef] [PubMed]

3. Givens, B.E.; Geary, S.M.; Salem, A.K. Nanoparticle-based CpG-oligonucleotide therapy for treating allergic asthma. Immunotherapy 2018, 10, 595-604. [CrossRef]

4. Kell, S.A.; Kachura, M.A.; Renn, A.; Traquina, P.; Coffman, R.L.; Campbell, J.D. Preclinical development of the TLR9 agonist DV281 as an inhaled aerosolized immunotherapeutic for lung cancer: Pharmacological profile in mice, non-human primates, and human primary cells. Int. Immunopharmacol. 2019, 66, 296-308. [CrossRef] [PubMed]

5. Brown, D.A.; Kang, S.H.; Gryaznov, S.M.; DeDionisio, L.; Heidenreich, O.; Sullivan, S.; Xu, X.; Nerenberg, M.I. Effect of phosphorothioate modification of oligodeoxynucleotides on specific protein binding. J. Biol. Chem. 1994, 269, 26801-26805. [CrossRef]

6. Sheehan, J.P.; Lan, H.-C. Phosphorothioate Oligonucleotides Inhibit the Intrinsic Tenase Complex. Blood 1998, 92, 1617-1625. [CrossRef]

7. Levin, A.A. A review of issues in the pharmacokinetics and toxicology of phosphorothioate antisense oligonucleotides. Biochim. Biophys. Acta. 1999, 1489, 69-84. [CrossRef]

8. Henry, S.P.; Beattie, G.; Yeh, G.; Chappel, A.; Giclas, P.; Mortari, A.; Jagels, M.A.; Kornbrust, D.J.; Levin, A.A. Complement activation is responsible for acute toxicities in rhesus monkeys treated with a phosphorothioate oligodeoxynucleotide. Int. Immunopharmacol. 2002, 2, 1657-1666. [CrossRef]

9. Tu, A.T.T.; Hoshi, K.; Ikebukuro, K.; Hanagata, N.; Yamazaki, T. Monomeric G-Quadruplex-Based CpG Oligodeoxynucleotides as Potent Toll-Like Receptor 9 Agonists. Biomacromolecules 2020, 21, 3644-3657. [CrossRef]

10. Hoshi, K.; Yamazaki, T.; Sugiyama, Y.; Tsukakoshi, K.; Tsugawa, W.; Sode, K.; Ikebukuro, K. G-quadruplex structure improves the immunostimulatory effects of cpg oligonucleotides. Nucleic Acid Ther. 2019, 29, 224-229. [CrossRef]

11. Liao, W.; Tan, M.; Kusamori, K.; Takakura, Y.; Nishikawa, M. Construction of Monomeric and Dimeric G-Quadruplex-Structured CpG Oligodeoxynucleotides for Enhanced Uptake and Activation in TLR9-Positive Macrophages. Nucleic Acid Ther. 2020, 30, 299-311. [CrossRef]

12. Hao, F.; Ma, Y.; Guan, Y. Effects of central loop length and metal ions on the thermal stability of G-quadruplexes. Molecules 2019, 24, 1863. [CrossRef] [PubMed]

13. Cheng, M.; Cheng, Y.; Hao, J.; Jia, G.; Zhou, J.; Mergny, J.L.; Li, C. Loop permutation affects the topology and stability of G-quadruplexes. Nucleic Acids Res. 2018, 46, 9264-9275. [CrossRef] [PubMed]

14. Ikebukuro, K.; Okumura, Y.; Sumikura, K.; Karube, I. A novel method of screening thrombin-inhibiting DNA aptamers using an evolution-mimicking algorithm. Nucleic Acids Res. 2005, 33, e108. [CrossRef]

15. Mazurov, A.V.; Titaeva, E.V.; Khaspekova, S.G.; Storojilova, A.N.; Spiridonova, V.A.; Kopylov, A.M.; Dobrovolsky, A.B. Characteristics of a new DNA aptamer, direct inhibitor of thrombin. Bull. Exp. Biol. Med. 2011, 150, 422-425. [CrossRef] [PubMed]

16. Spiridonova, V.A.; Barinova, K.V.; Glinkina, K.A.; Melnichuk, A.V.; Gainutdynov, A.A.; Safenkova, I.V.; Dzantiev, B.B. A family of DNA aptamers with varied duplex region length that forms complexes with thrombin and prothrombin. FEBS Lett. 2015, 589, 2043-2049. [CrossRef]

17. Russo Krauss, I.; Spiridonova, V.; Pica, A.; Napolitano, V.; Sica, F. Different duplex/quadruplex junctions determine the properties of anti-thrombin aptamers with mixed folding. Nucleic Acids Res. 2016, 44, 983-991. [CrossRef]

18. Mergny, J.L.; Lacroix, L. UV melting of G-quadruplexes. Curr. Protoc. Nucleic Acid Chem. 2009, 1-15. [CrossRef]

19. Hanagata, N.; Li, X.; Chen, M.H.; Li, J.; Hattori, S. Double-stranded phosphodiester cytosine-guanine oligodeoxynucleotide complexed with calcium phosphate as a potent vaccine adjuvant for activating cellular and Th1-type humoral immunities. Int. J. Nanomed. 2018, 13, 43-62. [CrossRef]

20. Honda, K.; Ohba, Y.; Yanai, H.; Negishi, H.; Mizutani, T.; Takaoka, A.; Taya, C.; Taniguchi, T. Spatiotemporal regulation of MyD88-IRF-7 signalling for robust type-I interferon induction. Nature 2005, 434, 1035-1040. [CrossRef]

21. Kejnovská, I.; Kypr, J.; Vorlíčková, M. Oligo(dT) is not a correct native PAGE marker for single-stranded DNA. Biochem. Biophys. Res. Commun. 2007, 353, 776-779. [CrossRef]

22. Largy, E.; Mergny, J.L.; Gabelica, V. Role of Alkali Metal Ions in G-Quadruplex Nucleic Acid Structure and Stability. In The Alkali Metal Ions: Their Role for Life, 1st ed.; Sigel, A., Sigel, H., Sigel, R., Eds.; Springer: Cham, Switzerland, 2016; Volume 16, pp. 203-258.

23. Duque, G.A.; Descoteaux, A. Macrophage cytokines: Involvement in immunity and infectious diseases. Front. Immunol. 2014, 5, 491. [CrossRef]

24. Noel, G.; Baetz, N.W.; Staab, J.F.; Donowitz, M.; Kovbasnjuk, O.; Pasetti, M.F.; Zachos, N.C. A primary human macrophageenteroid co-culture model to investigate mucosal gut physiology and host-pathogen interactions. Sci. Rep. 2017, 7, 45270. [CrossRef] [PubMed]

25. Lee, K.Y. M1 and M2 polarization of macrophages: A mini-review. Med. Biol. Sci. Eng. 2019, 2, 1-5. [CrossRef]

26. Germic, N.; Frangez, Z.; Yousefi, S.; Simon, H.U. Regulation of the innate immune system by autophagy: Monocytes, macrophages, dendritic cells and antigen presentation. Cell Death Differ. 2019, 26, 715-727. [CrossRef] [PubMed] 
27. van Dinther, D.; Veninga, H.; Iborra, S.; Borg, E.G.F.; Hoogterp, L.; Olesek, K.; Beijer, M.R.; Schetters, S.T.T.; Kalay, H.; GarciaVallejo, J.J.; et al. Functional CD169 on Macrophages Mediates Interaction with Dendritic Cells for CD8 + T Cell Cross-Priming. Cell Rep. 2018, 22, 1484-1495. [CrossRef] [PubMed] 